\title{
Effect of carbon assimilation on dry weight production and partitioning during vegetative growth of K-deficient cotton (Gossypium hirsutum L.) plants
}

Edward Gerardeaux • Etienne Saur •

Julie Constantin • Annabel Porté •

Lionel Jordan-Meille

Published online: 6 May 2009

(C) Springer Science + Business Media B.V. 2009

Erratum to: Plant Soil (2009) 324:329-343

DOI 10.1007/s11104-009-9950-Z

The original article was inadvertently published with an incomplete title. The complete title appears here.

Responsible Editor: Ismail Cakmak.

The online version of the original article can be found under doi:10.1007/s11104-009-9950-z.

E. Gerardeaux $(\bowtie) \cdot$ E. Saur $\cdot$ J. Constantin $\cdot$ A. Porté $\cdot$

L. Jordan-Meille

CIRAD,

Montpellier Cedex 5, France

e-mail: gerardeaux@cirad.fr

E. Saur

e-mail: e-saur@enitab.fr

J. Constantin

e-mail: julie.constantin@laon.inra.fr
A. Porté
e-mail: annabel@pierroton.inra.fr
L. Jordan-Meille
e-mail: 1-jordan@enitab.fr 